\title{
ON EXPONENTIALLY SMALL TERMS \\ OF SOLUTIONS TO NONLINEAR ORDINARY DIFFERENTIAL EQUATIONS
}

\author{
A. Tovbis
}

\begin{abstract}
The subject of the paper is exponential asymptotics, or in the other terminology, "asymptotics beyond all orders", to solutions of nonlinear ordinary differential equations. We present expressions for exponential corrections to the power series asymptotics of solutions under some generic assumptions on a given equation. We also discuss relations between exponential corrections and analytic properties of the Borel transform of the formal asymptotic series. In particular, we consider the Stokes phenomenon and show that the transition constant (the magnitude of the exponentially small "jump") is the same for all solutions which possess the same power series asymptotics in the considered region on the complex plane.
\end{abstract}

\section{Introduction}

We start the exposition with the following example. It is known (see [PRG], [GJ]) that the study of travelling wave solutions to the singularly perturbed fifth order Korteweg-de Vries equation

$$
\epsilon^{2} u_{x x x x x}+u_{x x x}+6 u u_{x}+u_{t}=0
$$

is reducible to the study of solutions of the ordinary differential equation

$$
v^{\prime \prime \prime \prime}(x)+v^{\prime \prime}(x)+v^{2}(x)=0,
$$

which possess the asymptotics

$$
v(x)=O\left(x^{-2}\right), \quad x \rightarrow \infty,
$$

in any closed proper subsector of the upper half-plane $\hat{S}$ of the complex $x$-plane.

Actually, the problem is to study the asymptotic behavior of $v(x)$ near the real semiaxes. It turns out that the corresponding asymptotics contain respectively exponential terms $c_{1} e^{i x}$ and $c_{2} e^{i x}$, where $c_{1,2} \in \mathbb{C}$. These terms are exponentially small in any proper subsector of $\hat{S}$, but become oscillatory and therefore "detectable" near the real semiaxes.

It is known that a solitary wave solution to (0.1) consists of the central core of the wave accompanied by co-propagating trailing oscillations. The constant $c_{2}-c_{1}$, which we call the transition constant of the equation (0.2) in $\hat{S}$, relates the amplitude and the phase shift of trailing oscillations and, therefore, is an important characteristic of the solitary wave solutions to $(0.1)$ ([GJ]).

The main results of the present paper, applied to the equation (0.2), are as follows:

Received July 20, 1993, revised October 29, 1993.

Supported by a grant of the Ministry of Science and Technology of Israel. 
a) The mappings $c_{1,2}: \Psi_{\hat{S}} \mapsto \mathbb{C}$ are global coordinates on the manifold $\Psi_{\hat{S}}$ of the solutions to $(0.2)$, satisfying $(0.3)$;

b) The transition constant $c=c_{2}-c_{1}$ does not depend on $v \in \Psi_{\hat{S}}$;

c) We present an explicit formula (see $\S 3.3$ for the equation (0.2) and (0.7) for the general case) for the transition constant $c$.

These results will be obtained for a system of nonlinear ordinary equations of the general type

$$
x^{1-r} z^{\prime}(x)=f(x, z), \quad x \in \overline{\mathbb{C}}, z \in \mathbb{C}^{n}, n, r \in \mathbb{N},
$$

which possesses a formal power series solution

$$
\hat{z}(x)=\sum_{k=n_{0}}^{\infty} z_{k} x^{-k / p}, \quad n_{0}, p \in \mathbb{N} .
$$

Here the vector-valued function $f(x, z)$ is holomorphic at $(\infty, 0) \in \overline{\mathbb{C}} \times \mathbb{C}^{n}$.

In fact the existence of the formal solution (0.5) follows from a condition of the type (0.3), namely (see [T2]) from

$$
x^{1-r} \hat{z}_{N}^{\prime}(x)-f\left(x, \hat{z}_{N}\right)=O\left(x^{-\frac{N+1}{p}}\right), \quad x \rightarrow \infty,
$$

where the number $N \in \mathbb{N}$ depends on the equation (0.4). (Here $\hat{z}_{N}$ denotes the first $N$ terms of (0.5)).

Once the formal solution (0.5) to (0.4) is fixed, one can define the characteristic exponentials of (0.4), related to (0.5) (see $\S 1.2$ ). For example, the second Painleve equation (3.10)

$$
v^{\prime \prime}(x)=2 v^{3}+x v+\alpha, \quad \alpha \in \mathbb{C}
$$

has two characteristic exponentials $x^{-1 / 4} e^{ \pm(2 / 3) x^{3 / 2}}$ related to the formal power series solution (3.11) with the leading term $-\alpha / x$. The other two characteristic exponentials $x^{-1 / 4} e^{ \pm 2 \sqrt{2 / 3} i x^{3 / 2}}$ are related to the other formal power series solutions with the leading terms $\pm \frac{i}{\sqrt{2}} x^{\frac{1}{2}}$ (see [T2], $\S 4.2$ ).

In this paper we consider the equation (0.4) of the generic type, i.e. satisfying the following assumptions:

a) The equation (0.4) is a one-level equation of some order $\nu$, i.e. the characteristic exponentials of (0.4) are of the order $\nu$ (see $\S 1.2)$;

b) There exists only one characteristic exponential, which is decreasing in the considered sector $\hat{S}$ of opening $\pi / \nu$.

In fact, this work is a generalization of [GM] to the case of nonlinear equations.

Let $\Psi_{S}$ denote the set of the solutions $z(x)$ of (0.4), which are holomorphic in every closed subsector of a given open sector $S$ on the Riemann surface of $\ln x$ for sufficiently large $|x|$, and which have the asymptotic expansion $\hat{z}(x)$ in every closed subsector of $S$. The latter fact is denoted by

$$
z(x) \sim \hat{z}(x), \quad x \rightarrow \infty, x \in S .
$$

It is known that $\Psi_{S}$ is nonempty if the opening of $S$ is less than $\pi / \nu$ ([RS]) and that either (0.6) is convergent (has a nonzero radius of convergence) or $\Psi_{S}=\emptyset$ when the opening of $S$ is sufficiently large. In fact, $\Psi_{S}$ is a complex analytic manifold, and the dimension of $\Psi_{S}$ is equal to the number of characteristic exponentials of (0.4), decreasing (in modulus) in every closed subsector of $S$ as $x \rightarrow \infty$ ([T2]). 
Let $\hat{S}$ denote a sector, where some characteristic exponential $e^{q(x)}$ of $(0.4)$ is decreasing as $x \rightarrow \infty$ and let $\lambda x^{\nu}$ be the leading term of $q(x)$. Then, according to the assumptions a) and b), the opening of $\hat{S}$ is equal to $\pi / \nu$ and the dimension of $\Psi_{\hat{S}}$ is equal to one. In $\S 1$ we show the existence of two different global coordinates $F_{1,2}: \Psi_{\hat{S}} \mapsto \mathbb{C}$. In fact, $F_{1,2}(z) h e^{q(x)}$, where $h \in \mathbb{C}^{n}$ is a certain constant vector and $\|h\|=1$, are the exponential terms which appear in the asymptotics of the solution $z \in \Psi_{\hat{S}}$ near the boundaries of $\hat{S}$. In the case $\nu=r$, for example, $h$ is the eigenvector of the Jacobian matrix $\frac{\partial f}{\partial z}(\infty, 0)$, corresponding to the eigenvalue $\lambda$. In $\S 2$ we show that the transition function $\varphi_{\hat{S}}=F_{2} \circ F_{1}^{-1}$ is given by $\varphi_{\hat{S}}(\alpha)=\alpha+c$, where $c \in \mathbb{C}$ is the transition constant. Usually by the Stokes phenomenon we understand the fact that $\varphi_{\hat{S}} \not \equiv i d$.

The characteristic exponential $e^{q(x)}$ of (0.4) and the corresponding vector $h$ could be derived from the equation ( 0.4$)$ by some algebraic operations. However the evaluation of the transition constant $c$ requires a principally different approach, since $c$ depends transcendentally on the coefficients of $f(x, z)$.

Generally, $q(x)$ is the sum of a polynomial of order $\nu$ and of a logarithmic term $b \ln x$, where $b \in \mathbb{C}$. However let us assume that $q(x)=\lambda x^{\nu}+b \ln x$. This is not a restrictive assumption since there exists a holomorphic in $x^{-\frac{1}{\nu}}$ change of the independent variable, reducing $q(x)-b \ln x$ to $\lambda x^{\nu}$. In $\S 3$ we show that

$$
c h=-2 \pi i \lim _{p \rightarrow \sqrt[\nu]{-\lambda}}\left(p^{\nu}+\lambda\right) \mathcal{L}_{\nu}^{-1}\left[x^{-b} z(x)\right](p),
$$

where $\mathcal{L}_{\nu}^{-1}$ is the inverse $\nu$-Laplace transform (see $\S 2$ ) of a certain solution $z \in \Psi_{\hat{S}}$. Here the constant $b$ and the vector $h$ are uniquely determined by (0.4). The choice of the root of $-\lambda$ depends on the sector $\hat{S}$.

Under the assumption a) the inverse $\nu$-Laplace transform $\mathcal{L}_{\nu}^{-1} z$ is holomorphic at the origin ([T3]). Therefore one can simply use the formal $\nu$-Borel transform $\mathcal{B}_{\nu}$ to construct $\mathcal{L}_{\nu}^{-1}\left[x^{-b} z(x)\right]$. This remark justifies the evaluation of Stokes constants via the $\nu$-Borel summation (which was utilized, for example, in [PRG], [GJ], [HM], etc.) in the case when $\mathcal{L}_{\nu}^{-1} z$ is holomorphic in the disk $|p|<|\lambda|^{\frac{1}{\nu}}$.

\section{Boundary behavior}

1.1. Prenormalization. In this subsection we derive the linear part of $(0.4)$, which determines the behavior of solutions at $x=\infty$ (see [T2]). In the following, for any square matrix $B, \operatorname{diag} B$ denotes the diagonal matrix with diagonal entries coinciding with those of $B$.

Let us consider the matrix linear differential equation

$$
x^{1-r} Y^{\prime}(x)=A(x) Y(x), \quad r \in \mathbb{N},
$$

where the matrix-valued function $A(x)$ is holomorphic at infinity.

Theorem 1.1 ([KT], [T1]). There exists some $p_{0} \in \mathbb{N}$, such that for any $\zeta \in \mathbb{N}$ the equation (1.1) is reducible to

$$
x^{1-r} Z^{\prime}(x)=\left[B(x)+o\left(x^{-\zeta}\right)\right] Z(x), \quad x \rightarrow \infty,
$$

by means of the transformation $Y(x)=T(x) Z(x)$. Here the matrix $B(x)$ (which does not depend on $\zeta)$ is upper-triangular and polynomial in $x^{-1 / p_{0}}$, and the order of $\operatorname{diag} B(x)$ does not exceed $r p_{0}$. The matrix $T(x)$ (depending on $\zeta$ ) is polynomial 
in $x^{-1 / p_{0}}$, $\operatorname{det} T(\infty) \neq 0$ and we can always assume that $\left\|T^{(1)}(\infty)\right\|=1$, where $T^{(j)}$ denotes the $j$-th column of the matrix $T$.

The equation (1.2) is called a triangular form of (1.1). Triangular forms of holomorphic matrix-valued functions with respect to similarity transformations were considered in [Fr]. Generally, the triangular form (1.2) is not uniquely determined. However it is easy to show (using, for instance, the theory of formal invariants of (1.1), see [BJL]) that the collection of diagonal entries $\left\{b_{i}(x)\right\}_{i=1}^{n}$ of $B(x)$ are uniquely determined modulo $O\left(x^{-r}\right)$. The following Corollary demonstrates the uniqueness of $\operatorname{diag} B(x)$ under a certain generic assumption.

Corollary 1.1. For all $j \geq 2$, let

$$
b_{1}(x) \not \equiv b_{j}(x) \bmod O\left(x^{-r}\right) .
$$

Then the first diagonal entry $b_{1}(x)$ of the triangular form (1.2), and the corresponding vector $T^{(1)}(\infty)$, are uniquely determined.

Proof. Suppose matrices $B(x)$ and $\tilde{B}(x)$ correspond to different triangular forms (1.2) of $(1.1)$, so that $b_{1}(x) \not \equiv \tilde{b}_{1}(x)$ but $b_{1}(x) \equiv \tilde{b}_{1}(x) \bmod O\left(x^{-r}\right)$. Then for any $\zeta$ there exists a polynomial in $x^{-1 / p_{0}}$ matrix $T(x)$, so that $\stackrel{\circ}{T}(\infty)$ is an invertible matrix and

$$
\stackrel{\circ}{T}(x) B(x)=\tilde{B}(x) \stackrel{T}{T}(x)-x^{1-r} \stackrel{\circ}{ }^{\prime}(x) .
$$

The equation for the $(n, 1)$-th entry of $(1.4)$ is

$$
t_{n, 1}^{\prime}(x)=x^{r-1}\left(\tilde{b}_{n}(x)-b_{1}(x)\right) t_{n, 1}(x)+o\left(x^{-\zeta+r-1}\right),
$$

where $t_{n, 1}$ denotes the $(n, 1)$-th entry of $\stackrel{\circ}{T}$. This differential equation may have a nontrivial polynomial in $x^{-1 / p_{0}}$ solution only if $\tilde{b}_{n}(x) \equiv b_{1}(x) \bmod O\left(x^{-r}\right)$. However, this contradicts (1.3) and our assumption. So, $t_{n, 1} \equiv 0$. By the same arguments we get $t_{j, 1} \equiv 0$ for all $j \geq 2$.

Let $\tilde{b}_{1}(x)-b_{1}(x)=\beta / x$, where $\beta \in \mathbb{C}$. Then for the $(1,1)$-th entry of $(1.4)$ we get $t_{1,1}^{\prime}=\beta t_{1,1} / x+o\left(x^{-\zeta+r-1}\right)$. The invertibility of $\stackrel{\circ}{T}(\infty)$ implies $t_{1,1}(\infty) \neq 0$. One can easily check that $\beta=0$ is the necessary condition for $t_{1,1}(\infty) \neq 0$. So, $\tilde{b}_{1}(x) \equiv b_{1}(x)$.

Let $T(x)$ and $\tilde{T}(x)$ denote the matrices which reduce (1.1) respectively to the triangular forms, considered above. Then $T(x)=\tilde{T}(x) \dot{T}(x)$. The vector $T^{(1)}(\infty)$ is parallel to $\tilde{T}^{(1)}(\infty)$, since all the off-diagonal entries of $\tilde{T}^{(1)}(x)$ are equal to zero but $\stackrel{\circ}{T}^{(1)}(\infty) \neq 0$. Now the fact that $\left\|T^{(1)}(\infty)\right\|=\left\|\tilde{T}^{(1)}(\infty)\right\|=1$ completes the proof.

The following example shows that one cannot omit the condition (1.3) in Corollary 1.1 .

Example 1.1. The differential equation

$$
Y^{\prime}(x)=\left(\begin{array}{cc}
0 & 1 \\
x^{-2} & 0
\end{array}\right) Y(x)
$$

has two different triangular forms (1.2), where

$$
B(x)=\left(\begin{array}{cc}
\alpha / x & 1+O\left(x^{-1}\right) \\
0 & -\alpha / x
\end{array}\right) .
$$

Here $\alpha$ is either of the two roots of the quadratic equation $\alpha(\alpha-1)-1=0$. 
In order to simplify the following exposition (and without any loss of generality) we will assume both in Theorem 1.1 and in the formal solution (0.5) of (0.4) that the denominators $p, p_{0}$ are equal to one.

Let deg of a series in negative powers of $x$ denote the exponent of the highest power of $x$ occurring in the series; we set $\operatorname{deg} a(x)=-\infty$ if $a(x) \equiv 0$. Let us denote $\eta=2 n(r+1)+\delta-1$, where $\delta \in \mathbb{Z}^{+}$is to be defined below. Then by the change of variables $z \mapsto z+\sum_{n_{0}}^{\eta} z_{k} x^{-k}$ with $z_{k}$ as in (0.5), we obtain the inequality

$$
\operatorname{deg} f(x, 0) \leq-(\eta+1)
$$

for the new free term $f(x, 0)$ of $(0.4)$. Now by means of Theorem 1.1 we can assume that the Jacobian matrix $\left.\frac{\partial f}{\partial z}\right|_{z=0}$ is in the triangular form $B(x)+o\left(x^{-\zeta}\right)$, where we set $\zeta=(n-1)(r+1)+1$. This does not affect $\operatorname{deg} f(x, 0)$ since $T(\infty)$ is invertible.

The last step of our reduction is given by the so-called "shearing transformation" $z(x)=S(x) y(x)$ (see [Wa], §19), where

$$
S(x)=\operatorname{diag}\left(x^{-n(r+1)-\delta}, \ldots, x^{-(2 n-1)(r+1)-\delta}\right) .
$$

Then the equation (0.4) is reduced to

$$
y^{\prime}=P(x) y+\tilde{f}(x, y)
$$

and one can directly check that:

a) $\tilde{f}(x, y)$ is holomorphic at $(\infty, 0) \in \overline{\mathbb{C}} \times \mathbb{C}^{n}$;

b) $\operatorname{deg} \tilde{f}(x, 0) \leq-2$;

c) $\left.\operatorname{deg} \frac{\partial \tilde{f}}{\partial y}\right|_{y=0} \leq-2$;

d) the coefficient of any higher order monomial (in entries of the vector $y$ ) of $\tilde{f}(x, y)$ has degree not more than $-2-\delta$

e) $x P(x)$ is a diagonal matrix which is polynomial in $x$ of order not more than $r$. The free term of $x P(x)$ is equal to $B_{r}+[n(r+1)+\delta] I+\operatorname{diag}(0, r+$ $1, \ldots,(n-1)(r+1))$, where $I$ is the identity matrix and $B_{r}=\operatorname{diag}\left(b_{1}, \ldots, b_{n}\right)$ is the leading coefficient of the polynomial (in $x^{-1}$ ) $\operatorname{diag} B(x)$. We choose the minimal $\delta \in \mathbb{Z}^{+}$, such that the entries of the free term of $x P(x)$ have nonnegative real parts.

The equation (1.5) is called a prenormalized form of the equation (0.4). In what follows we use the notation $\Psi_{S}$, whenever it does not lead to any confusion, to denote the corresponding sets of solutions to both the original and the prenormalized equations.

1.2. Volterra-type integral equations. The equation (1.5) can be considered as a perturbation of the linear equation $y^{\prime}=P(x) y$ and hence can be written as

$$
y(x)=e^{Q(x)}\left(C+\int_{\Gamma(x)} e^{-Q(t)} \tilde{f}(t, y) d t\right)
$$

where $Q^{\prime}(x)=P(x), C \in \mathbb{C}^{n}$ and $\Gamma(x)$ is a collection of $n$ individual contours of integration for each entry of the vector integrand. The contours $\Gamma^{j}(x)$ of $\Gamma(x)$ have the common endpoint $x$.

The entries $q_{j}(x)$ of the diagonal matrix $Q(x)$ consist of polynomial and logarithmic parts. Let $J$ denote the set of indicies $j$, such that $q_{j}(x)$ has a non-trivial polynomial part, and let $\lambda_{j} x^{\nu_{j}}$, where $\lambda_{j} \in \mathbb{C} \backslash\{0\}$ and $\nu_{j} \in\{1, \ldots, r\}$, denote the leading term of $q_{j}$. 
Definition 1.1. The exponentials $e^{q_{j}(x)}$, where $j \in J$, are called characteristic exponentials of the equation (0.4).

Definition 1.2. A ray $\tau$, issuing from the origin, is called a Stokes ray of the equation (0.4) if $\Re\left(\lambda_{j} x^{\nu_{j}}\right)=0$ when $x \in \tau$.

Let $S$ be a sector of opening less than $\pi / \nu$, where $\nu=\max _{j \in J} \nu_{j}$, in the complex plane. We assume the boundary rays of $S$ are not Stokes rays of (0.4). With any such $S$ in [T2] we associate the subspace $L_{S} \in \mathbb{C}^{n}$ and the collection of contours $\Gamma_{S}(x)=\left\{\Gamma_{S}^{j}(x)\right\}_{j=1}^{n}$ (which may depend on $C \in L_{S}$, see below), so that the following statement is true.

Theorem 1.2 ([T2]). a) The set of solutions of (1.6), where $C \in L_{S}$ and $\Gamma(x)=$ $\Gamma_{S}(x)$, coincides with $\Psi_{S}$;

b) The dimension of the manifold $\Psi_{S}$ is equal to the number of characteristic exponentials decreasing in $S$ as $x \rightarrow \infty$;

c) The equation (1.6), where $\Gamma(x)=\Gamma_{S}(x)$ and $C \in L_{S}$ are fixed, has a unique solution $y(x)$. This solution can be represented as

$$
y(x)=\sum_{j=1}^{\infty} w_{j}(x)
$$

where $w_{j}(x)=y_{j}(x)-y_{j-1}(x), y_{0}(x) \equiv 0$ and

$$
y_{j}(x)=e^{Q(x)}\left(C+\int_{\Gamma_{S}(x)} e^{-Q(t)} \tilde{f}\left(t, y_{j-1}(t)\right) d t\right) .
$$

The sum (1.7) converges uniformly and absolutely in the sufficiently remote part of $S$.

The assumption a) from the Introduction means that $J \neq \emptyset$ and $\nu_{j}=\nu$ for all $j \in J$. According to the assumption b) and without any loss of generality we can assume that the exponential $e^{\lambda_{1} x^{\nu}}$ is the only exponential from among the $e^{\lambda_{j} x^{\nu}}(j \in J)$ decreasing in the sector $\hat{S}=\left\{x: 0<\arg x<\frac{\pi}{\nu}\right\}$. By $\stackrel{\circ}{S}$ and $\tilde{S}$ we denote small perturbations of $\hat{S}$, such that $\stackrel{S}{S}$ is a proper subsector of $\hat{S}$ and that $\tilde{S}$ contains the ray $\arg x=0$. We also assume that the opening of $\tilde{S}$ is less than $\pi / \nu$, that $\tilde{S} \supset \stackrel{\circ}{S}$ and that no Stokes rays are contained in $\hat{S} \backslash \stackrel{\circ}{S}$.

For any $x_{0} \in S$ we introduce a closed subregion

$$
S_{x_{0}}=\Pi_{\nu}^{-1} \circ \Sigma_{x_{0}} \circ \Pi_{\nu}(S)
$$

where the transformations $\Pi_{u}, u>0$, and $\Sigma_{\xi}, \xi \in \mathbb{C}$, are defined by

$$
\Pi_{u}: x \mapsto x^{u} ; \quad \Sigma_{\xi}: x \mapsto x+\xi .
$$

For an arbitrary point $x \in \tilde{S}$ the $j$-th contour $\tilde{\Gamma}^{j}(x)$ of the collection $\tilde{\Gamma}(x)=\Gamma_{\tilde{S}}(x)$ is defined as a ray, issuing from $x$, such that $\tilde{\Gamma}^{j}(x) \subset \tilde{S}_{x_{0}}$ and $e^{-q_{j}(t)}$ is decreasing along $\tilde{\Gamma}^{j}(x)$. In this case $L_{\tilde{S}}=\{0\}$, and (1.6) has a unique solution. It is clear that the directions of contours $\tilde{\Gamma}^{j}(x)$ can be slightly changed preserving the values of corresponding integrals. Then, since the opening of $\tilde{S}$ is less then $\pi / \nu$, one can directly show the existence of some $\sigma>0$, such that for any $x_{0} \in \tilde{S}, x \in \tilde{S}_{x_{0}}$ and any $t \in \tilde{\Gamma}^{j}(x), j=1, \ldots, n$

$$
|x|>\sigma\left|x_{0}\right|, \quad|t|>\sigma|x|
$$


Let now $x_{0} \in \stackrel{\circ}{S}, x \in \stackrel{\circ}{S}_{x_{0}}$. Then we define the collection $\stackrel{\circ}{\Gamma}(x)$ as coinciding with $\tilde{\Gamma}(x)$ except for the contour $\stackrel{\circ}{\Gamma}^{1}(x)$. (Note that we can deform any contour $\tilde{\Gamma}^{j}(x), j>1$ so that $\tilde{\Gamma}^{j}(x) \subset \stackrel{\circ}{S}_{x_{0}}$.) The exponential $e^{-q_{1}(x)}$ is increasing in the whole $\hat{S}$, and therefore $\tilde{\Gamma}^{1}(x)$ is to be a finite contour. We choose it as the segment $\left[x_{0}, x\right]$ (see [Wa], §14.3 for details). The subspace $L_{S}$ is spanned by the first coordinate vector. According to the theorem of analytic dependence on the initial data and to the assertion a) of Theorem 1.2 , the first entry $C_{(1)}$ of the vector $C$ is a local coordinate on the manifold $\Psi_{S}$ in a neighborhood of the solution to the corresponding equation (1.6).

1.3. Boundary curves. The objective of $\S 1$ is to study the behavior of solutions of $\Psi_{S}$ on the boundary Stokes ray $\tau$. The following simple example illustrates some difficulties related to this problem.

The functions $y(x) \equiv \pm i$ and $y(x)=\tan (x+c)$, where $c \in \mathbb{C}$, represent all solutions of the scalar equation $y^{\prime}(x)=1+y^{2}(x)$ of order $\nu=1$. Any solution $y(x)$, except $y(x) \equiv-i$, admits the asymptotic expansion

$$
y(x) \sim i, \quad x \rightarrow \infty, x \in \hat{S}
$$

so it belongs to $\Psi_{S_{S}}$. However in the case $c \in \mathbb{R}$ the function $\tan (x+c)$ has infinitely many poles, which are accumulating to $x=\infty$, on both Stokes rays $\arg x=0$ and $\arg x=\pi$.

This example shows that generally a solution $y \in \Psi_{S}$ may have very complicated behavior on the boundary Stokes ray $\arg x=0$. Granting this fact, our approach is to study the asymptotics of $y \in \Psi_{S}$ along a certain curve $\ell$, such that $\arg x \rightarrow 0$ as $|x| \rightarrow \infty, x \in \ell$.

For any given $M>0, \mu \geq 0$ we define the curve $\ell=\ell(\mu, M)$ by the equation

$$
\left|e^{q_{1}(x)}\right|=M|x|^{-\mu}
$$

where $\arg x$ is assumed to be bounded. Let us represent

$$
q_{1}(x)=\lambda_{1} x^{\nu}+\sum_{k=1}^{\nu-1} \alpha_{k} e^{i \theta_{k}} x^{\nu-k}+a_{\nu} \ln x
$$

where $\alpha_{k} \geq 0,0 \leq \theta_{k}<2 \pi, a_{\nu} \in \mathbb{C}$. Moreover, $\lambda_{1}=i\left|\lambda_{1}\right|$ since $e^{q_{1}(x)}$ is decreasing in $\hat{S}$. Then (1.10) can be represented as $\Re q_{1}(x)=\ln M-\mu \Re \ln x$ or

$$
-\left|\lambda_{1}\right| \rho^{\nu} \sin \nu \phi+\sum_{k=1}^{\nu-1} \alpha_{k} \rho^{\nu-k} \cos \left((\nu-k) \phi+\theta_{k}\right)+\left(\Re a_{\nu}+\mu\right) \ln \rho-\phi \Im a_{\nu}-\ln M=0
$$

where $x=\rho e^{i \phi}$. The assumption that $\phi$ is bounded implies $\lim _{\rho \rightarrow \infty} \phi=0$. Then for sufficiently large $\rho$ we get

$$
\phi=\frac{\sum_{k=1}^{\nu-1} \alpha_{k} \rho^{\nu-k} \cos \theta_{k}+\left(\Re a_{\nu}+\mu\right) \ln \rho-\ln M}{\left|\lambda_{1}\right| \nu \rho^{\nu}+\sum_{k=1}^{\nu-1}(\nu-k) \alpha_{k} \rho^{\nu-k} \sin \theta_{k}+\Im a_{\nu}}
$$

which implies $\phi=O\left(\rho^{-1}\right)$ as $\rho \rightarrow \infty$. (Moreover, if all the coefficients of the polynomial part of $q_{1}(x)$ are purely imaginary, then $\phi=O\left(\frac{\ln \rho}{\rho^{\nu}}\right)$ as $\rho \rightarrow \infty$.) 
1.4. Behavior of solutions along $\ell$. Let $S$ denote the part of $\tilde{S}$ which is bounded by $\ell=\ell(M, \mu)$ from below (we assume $\ell \subset S), x_{0} \in S$ and $S_{x_{0}}=\tilde{S}_{x_{0}} \cup S$. Then for any $x \in S_{x_{0}}$ the collection of contours $\Gamma_{S}(x)$ is defined as coinciding with $\tilde{\Gamma}(x)$ except for the first contour $\Gamma_{S}^{1}(x)$. This contour is chosen as the union of: the segment of $\tilde{\Gamma}^{1}(x)$ from the point $x$ to the point $\xi(x)=\tilde{\Gamma}^{1}(x) \cap \ell$; the part of the curve $\ell$ from $\xi(x)$ to infinity. The following Theorem 1.3 is in a sense an extension of Theorem 1.2 to the curvilinear sector $S$.

Theorem 1.3. For any $M>0$ and $\mu \in(0,1)$ the equation (1.6), where $\Gamma(x)=\Gamma_{S}(x)$ and $C \in L_{\dot{S}}$ is fixed, has a unique solution $y(x)$, which possesses the asymptotics

$$
y(x)-e^{Q(x)} C=O\left(x^{-1}\right), \quad x \rightarrow \infty, x \in S .
$$

Moreover, this solution can be represented by (1.7), (1.8). For any sufficiently remote point $x_{0} \in S$ the sum (1.7) converges uniformly and absolutely in $S_{x_{0}}$.

Proof. The properties of the integral operator, occurring in (1.6), were studied in [Wa], $\S 14$. Using Lemma 14.2 from there and the property $(1.5), \mathrm{b})$, we get

$$
\left\|w_{1}(x)\right\|<B|x|^{-\mu}, \quad x \in S_{x_{0}},
$$

for some $x_{0} \in S$, where $\left|x_{0}\right|$ is a sufficiently large number. Here $w_{j}(x), j \in \mathbb{N}$, are defined by (1.8) and $B=2 M(\|C\|)$.

The assertion of the theorem is a consequence of the estimate

$$
\left\|w_{j+1}(x)\right\| \leq \frac{K}{|x|}\left\|w_{j}(x)\right\|, \quad x \in S_{x_{0}}
$$

which holds for some $K, x_{0} \in S$ and all $j \in \mathbb{N}$. We prove (1.12) by induction using the properties of $\tilde{f}$ and the following known statement.

Statement 1.1. Let $h(x): \mathbb{C}^{n} \mapsto \mathbb{C}^{n}$ be a holomorphic mapping in a neighborhood of $0 \in \mathbb{C}^{n}$ and let $h(x)$ contain no constant and linear terms. Then there exists a disk $V_{\beta}=\left\{x \in \mathbb{C}^{n}:\|x\|<\beta\right\}$, where $\beta>0$, and a matrix-valued function $H(y, z): V_{\beta}^{2} \mapsto \mathbb{C}^{n^{2}}$ so that $H(y, z)$ is holomorphic in $V_{\beta}^{2}$ and for any $y, z \in V_{\beta}$

$$
h(y)-h(z)=H(y, z)(y-z) .
$$

Moreover $\|H(y, z)\| \rightarrow 0$ as $\beta_{1} \rightarrow 0$ uniformly for all $y, z \in V_{\beta_{1}}$, where $\beta_{1}<\beta$.

Let $k \in \mathbb{N}$ and let (1.12) hold for any positive integer $j<k$. Without any loss of generality we can assume that $\left|x_{0}\right|>2 K \sigma^{-1}$, so $\left\|y_{j}(x)\right\|<2 B|x|^{-\mu}$ for any $x \in S_{x_{0}}$. Then by virtue of Statement 1.1 the estimate

$$
\left\|\tilde{f}\left(x, y_{j}\right)-\tilde{f}\left(x, y_{j-1}\right)\right\| \leq \frac{K_{1}}{\left|x^{2}\right|}\left\|w_{j}\right\|, \quad j \leq k, x \in S_{x_{0}}
$$

follows from the properties (1.5), a)-d). Here the constant $K_{1}>0$ depends on $B$ but does not depend on $\left|x_{0}\right|$, as $\left|x_{0}\right| \rightarrow \infty$ and $\arg x_{0}=$ const.

Now, to get the estimate (1.12) for $j=k$ one needs to combine (1.13) with Lemma 14.2, [Wa]. Let us however note that the integral along $\Gamma_{S}^{1}(x)$ needs a special consideration since it is not the subject of Lemma 14.2. According to (1.10) and (1.13) the integral along $\ell$ from $\xi(x)$ to $\infty$ times $e^{q_{1}(x)}$ does not exceed

$$
\frac{2 K_{1}}{1-\mu} \frac{\left\|w_{j}(x)\right\|}{|\xi(x)|}
$$


This remark together with (1.9) completes the proof.

Remark 1.1. Theorem 1.3 is true under various weakenings of the restrictions a), b) from the Introduction. For instance, a) can be simply dropped. However this causes a considerable complication of the proof.

Remark 1.2. For a given $\mu \in(0,1)$ the constant $K=K(B)$ in (1.12) is completely determined by $B$. So, the estimate (1.12) is uniform for equations (1.6), where $\Gamma(x)=$ $\Gamma_{S}(x), C \in L_{S}$ and $\|C\| \leq \frac{B}{2 M}$.

Remark 1.3. Let us represent the vector $C$ from (1.6) as the sum of some absolutely convergent series $C=\sum_{j=1}^{\infty} C_{j}$. We can introduce $\tilde{w}_{j}=\tilde{y}_{j}-\tilde{y}_{j-1}$, where $\tilde{y}_{0} \equiv 0$ and

$$
\tilde{y}_{j}=e^{Q(x)}\left[\sum_{k=1}^{j} C_{k}+\int_{\Gamma(x)} e^{-Q(t)} \tilde{f}\left(t, \tilde{y}_{j-1}(t)\right) d t\right], \quad j>1 .
$$

Then the series $\sum_{j=1}^{\infty}\left\|\tilde{w}_{j}(x)\right\|$ converges uniformly in $S_{x_{0}}$ for some sufficiently remote point $x_{0} \in S$, and their sum is equal to (1.7). Moreover $\sum_{j=1}^{\infty} w_{j}(x) \equiv \sum_{j=1}^{\infty} \tilde{w}_{j}(x)$. The corresponding assertion can be extended to an arbitrary contractive operator in a Banach space.

Remark 1.4. The contour $\Gamma_{S}^{1}(x)$ is defined via the boundary curve $\ell(\mu, M)$. However for a given $C \in L_{S}$ the solution of (1.6) does not depend on the particular choices of $M>0$ and $\mu \in(0,1)$. Indeed, according to (1.12) the variation of $\Gamma_{S}^{1}(x)$, caused by the corresponding variation of $M$ and $\mu$, does not affect the terms $w_{j}(x)$ of (1.7) (but may increase the corresponding value of $\left|x_{0}\right|$ ).

Theorem 1.4. The set of solutions of (1.6), where $\Gamma(x)=\Gamma_{S}(x)$ and $C \in L_{\dot{S}}$, coincides with $\Psi_{\dot{S}}$.

Proof. Let $y(x)$ satisfy (1.6) for some $C \in L_{S}$ and $\Gamma(x)=\Gamma_{S}(x)$. Then $y(x)$ is also the solution of (1.6), where $\Gamma(x)=\stackrel{\circ}{\Gamma}(x)$ and $C=\tilde{C} \in L_{S}$. Here the collection of contours $\stackrel{\circ}{\Gamma}(x)$ is determined by some sufficiently remote point $x_{0} \in \stackrel{\circ}{S}$, and

$$
\tilde{C}_{(1)}=C_{(1)}+\int_{\Gamma_{S}^{1}\left(x_{0}\right)} e^{-q_{1}(t)} \tilde{f}_{(1)}(t, y) d t
$$

where a subindex in brackets here and henceforth means the corresponding entry of a vector. Hence, according to Theorem $1.2, y(x) \in \Psi_{\dot{S}}$.

To prove the opposite inclusion let us assume that $y(x)$ is the solution of (1.6), where $\Gamma(x)=\stackrel{\circ}{\Gamma}(x)$ and $C=\tilde{C} \in L_{\dot{S}}$. Then, according to Theorem $1.2, y(x)$ can be formally represented as (1.7), where

$$
y_{j}(x)=e^{Q(x)}\left(\left[\tilde{C}+\int_{\dot{\Gamma}(x)-\Gamma_{S}(x)} e^{-Q(t)} \tilde{f}\left(t, y_{j-1}\right) d t\right]+\int_{\Gamma_{S}(x)} e^{-Q(t)} \tilde{f}\left(t, y_{j-1}\right) d t\right)
$$

or as $(1.14)$, where $\tilde{y}_{j}=y_{j}$ and

$$
C_{k}=\int_{\dot{\Gamma}(x)-\Gamma_{\mathcal{S}}(x)} e^{-Q(t)}\left\{\tilde{f}\left(t, \tilde{y}_{k-1}\right)-\tilde{f}\left(t, \tilde{y}_{k-2}\right)\right\} d t, \quad k>1 .
$$


So, according to Remark 1.3, it remains to show the existence of the integrals (1.17) and the convergence of $\sum_{k=1}^{\infty} C_{k}$, where

$$
C_{1}=\tilde{C}+\int_{\dot{\Gamma}(x)-\Gamma_{S}(x)} e^{-Q(t)} \tilde{f}(t, 0) d t .
$$

Let $B=2 M\|\tilde{C}\| \sigma^{-\mu}$ and $K=K(B)$, and let $\left|x_{0}\right|$ be so large that

$$
\frac{K}{\left|x_{0}\right|} \chi \leq \frac{1}{2}
$$

where $\chi=1+\sigma^{-1}$. Under these assumptions, by induction we can easily prove

$$
\left\|\tilde{w}_{j+1}(x)\right\| \leq\left(\frac{K}{\left|x_{0}\right|} \chi\right)^{j} B\left|x_{0}\right|^{-\mu}, \quad j \in \mathbb{N}, x \in S_{x_{0}},
$$

where the $\tilde{w}_{j}$ are defined in Remark 1.3. Indeed, by virtue of (1.9), in the same way as in Theorem 1.3 we get

$$
\left\|\tilde{w}_{1}(x)\right\| \leq B\left|x_{0}\right|^{-\mu} .
$$

Let now $k \in \mathbb{N}$ and let (1.20) be true for all $j<k$. Then (1.19) implies $\left\|\tilde{y}_{j}(x)\right\| \leq$ $2 B\left|x_{0}\right|^{-\mu}$ for any $x \in S_{x_{0}}$. Therefore the estimate (1.12) is applicable to both integrals in (1.16), so

$$
\left\|\tilde{w}_{k+1}(x)\right\| \leq \frac{K}{\left|x_{0}\right|}\left\|\tilde{w}_{k}\left(x_{0}\right)\right\|+\frac{K}{|x|}\left\|\tilde{w}_{k}(x)\right\| .
$$

The inequality (1.20) for $j=k$ and thus for all $j \in \mathbb{Z}^{+}$follows from (1.9), (1.21), (1.22) and the inductive hypothesis.

The estimate (1.20) implies the existence of the integrals in (1.16) and the convergence of $\sum_{k=1}^{\infty} C_{k}$.

Let us return to the assumption (1.19). The solution $y(x)$ of $(1.6)$, where $\Gamma(x)=$ $\stackrel{\circ}{\Gamma}(x)$ and $C=\tilde{C} \in L_{\dot{S}}$, satisfies $e^{-q_{1}\left(x_{0}\right)} y_{(1)}\left(x_{0}\right)=\tilde{C}_{(1)}$. Hence, the constant $\tilde{C}_{(1)}=$ $\tilde{C}_{(1)}\left(x_{0}\right)$ may increase exponentially as the point $x_{0}$ is directed to infinity within $\stackrel{S}{S}$, and therefore may cause the corresponding increase of $K(B)$. To avoid this difficulty let us vary the curve $\ell=\ell\left(M\left(x_{0}\right), \mu\right)$, where the constant $M\left(x_{0}\right)$ is determined by $M\left(x_{0}\right)\left\|\tilde{C}\left(x_{0}\right)\right\|=B$.

A point $x_{0} \in \stackrel{\circ}{S}$ belongs to the sector $S$, determined by the curve $\ell\left(M\left(x_{0}\right), \mu\right)$, if and only if $\left|e^{q_{1}\left(x_{0}\right)}\right| \leq M\left(x_{0}\right)\left|x_{0}\right|^{-\mu}$. This inequality is equivalent to $\left\|y_{(1)}\left(x_{0}\right)\right\| \cdot\left|x_{0}\right|^{\mu} \leq B$, which for any sufficiently remote point $x_{0} \in \stackrel{\circ}{S}$ follows from (1.11). So $y(x)$ satisfies (1.6), where the contour $\Gamma_{S}^{1}(x)$ is determined by the corresponding curve $\ell\left(M\left(x_{0}\right), \mu\right)$. It follows now that Remark 1.4 completes the proof.

\section{Transition function}

2.1. Stokes phenomenon for nonlinear equations. Let us consider a sector $\hat{S}$, bounded by two successive Stokes rays $\tau_{1}$ and $\tau_{2}$, which corresponds to the exponential $e^{q_{1}(x)}$. Let $e^{q_{1}(x)}$ be decreasing in $\hat{S}$ and let the curves $\ell_{1}$ and $\ell_{2}$ be defined by (1.10), where $M>0$ and $\mu \in(0,1)$, with respect to the rays $\tau_{1}$ and $\tau_{2}$ correspondingly. 
Corollary 2.1. There exist two one-to-one correspondences $\hat{F}_{j}: \Psi_{\hat{S}} \mapsto L_{\hat{S}}, j=1,2$, such that

$$
y(x)=e^{Q(x)} \hat{F}_{j}(y)+O\left(x^{-1}\right), \quad x \rightarrow \infty, x \in S_{j} .
$$

Here $S_{1,2}$ is a closed sectorial region, bounded by $\ell_{1,2}$ from below (above) and by an arbitrary inner ray of $\hat{S}$ from above (below).

This corollary is a direct consequence of Theorems 1.3 and 1.4. Let $F_{j}=P \hat{F}_{j}$, where $P: \mathbb{C}^{n} \mapsto L_{\hat{S}}$ is the projector orthogonal to all but the first coordinate vectors of $\mathbb{C}^{n}$. The functions $F_{j}$ can be considered as global coordinates on $\Psi_{\hat{S}}$, i.e. they present "natural" parametrizations of $\Psi_{\hat{S}}$.

Let us define the transition function $\varphi_{\hat{S}}: \mathbb{C} \rightarrow \mathbb{C}$ by $\varphi_{\hat{S}}=F_{2} \circ F_{1}^{-1}$. It is possible to show that the fact that $\varphi_{\hat{S}} \equiv i d$ for all $e^{q_{j}}, j \in J$ in all corresponding sectors $\hat{S}$ is equivalent to the convergence of the formal power series solution (0.5) of (0.4).

Statement 2.1. The transition function $\varphi_{\hat{S}}(c)$ is a linear function.

Proof. Let us first show the analyticity of $F_{j}^{ \pm 1}, j=1,2$, at an arbitrary point $c \in \mathbb{C}$. For $F_{j}^{-1}(c)$ this fact can be shown by applying Theorem 1.3 to the equation (1.6), after (1.6) has been differentiated with respect to the parameter $c=C_{(1)}$. The analyticity of $F_{j}$ follows from the analyticity and univalence of $F_{j}^{-1}$ (we consider the local coordinate on $\Psi_{\hat{S}}$ given by (1.6) with some $x_{0} \in \stackrel{\circ}{S}, \Gamma(x)=\stackrel{\circ}{\Gamma}(x)$, see $\S 1$ for notations). Then the statement follows from Corollary 2.1 and from the fact that linear functions are the only entire schlicht functions.

The free term $c$ of the transition function

$$
\varphi_{\hat{S}}(z)=a z+c
$$

is called the transition constant of (1.5) in the sector $\hat{S}$. In this section we show that $a=1$ and give an explicit formula for the transition constant $c$.

2.2. Evaluation of $c$ by means of the inverse Laplace transforms. Let $\lambda_{j} x^{\nu}$ be the leading terms of $q_{j}(x), j \in J$, and let $\sqrt[\nu]{-\lambda_{j}}=\left|\lambda_{j}\right|^{1 / \nu} e^{\frac{i}{\nu}\left(\arg \lambda_{j}-\pi\right)}$. By $U$ we denote the complex $p$-plane with the cuts from points $\sqrt[\nu]{-\lambda_{j}} e^{\frac{2 \pi i k}{\nu}}, j \in J, k=$ $0, \ldots, \nu-1$, in the directions $\arg p=\frac{1}{\nu}\left(\arg \lambda_{j}+(2 k-1) \pi\right)$ respectively.

In what follows we use the standard direct and inverse $\nu$-Laplace transforms defined by the formulas

$$
\begin{aligned}
& \left(\mathcal{L}_{\nu} Y(p)\right)(x)=\nu \int_{0}^{\infty} e^{-x^{\nu} p^{\nu}} p^{\nu-1} Y(p) d p \\
& \left(\mathcal{L}_{\nu}^{-1} y(x)\right)(p)=\frac{\nu}{2 \pi i} \int_{\gamma_{0}} e^{p^{\nu} x^{\nu}} x^{\nu-1} y(x) d x
\end{aligned}
$$

respectively. Here the contour $\gamma_{0}=\Pi_{1 / \nu}(\tilde{\gamma})$, where $\tilde{\gamma}$ is a vertical line in the right halfplane and $\Pi_{u}(x)=x^{u}$. Note that $\mathcal{L}^{ \pm 1}=\mathcal{L}_{1}^{ \pm 1}$ are the usual direct and inverse Laplace transforms.

Let $\tilde{S}$ be an arbitrary sector with opening more than $\pi / \nu$ and $y \in \Psi_{\tilde{S}}$. Then by $\mathcal{L}_{\nu}^{-1} y$ we mean the integral (2.4) along the contour $\gamma_{0}$, turned around the origin in such a way that $\gamma_{0} \subset \tilde{S}$. 
Theorem 2.1 ([T3]). Let (0.4) be a one-level equation of the order $\nu$ and $y \in \Psi_{\tilde{S}}$. Then $Y(p)=\left[\mathcal{L}_{\nu}^{-1} y\right](p)$ is holomorphic in $U$.

Remark 2.1. The fact that $Y(p)$ is holomorphic at $p=0$ implies $Y(p)=\left[\mathcal{B}_{\nu} \tilde{y}\right](p)$, where $\mathcal{B}_{\nu}$ is the formal $\nu$-Borel transform. Hence $Y(p)$ does not depend on the choice of $\tilde{S}$.

Remark 2.2. In what follows for the sake of simplicity we assume that $q_{1}(x)=$ $\lambda_{1} x^{\nu}+d \ln x$, where $d=b_{1}+n(r+1)+\delta$. This is a non-restrictive assumption which can always be achieved by an appropriate change of the independent variable $x$.

Theorem 2.2. Let (1.5) be a prenormalized form of the one level equation (0.4) of order $\nu$, and let the characteristic exponential $e^{q_{1}(x)}$ be decreasing in the sector $\hat{S}$ of opening $\frac{\pi}{\nu}$. Then the corresponding transition constant can be given as

$$
c=-2 \pi i \lim _{p \rightarrow \sqrt[\nu]{-\lambda_{1}}}\left(p^{\nu}+\lambda_{1}\right) \stackrel{\circ}{(1)}_{(p)},
$$

where $\stackrel{\circ}{Y}_{(1)}(p)=\left[\mathcal{L}_{\nu}^{-1} x^{-d} y_{(1)}(x)\right](p)$, and the limit in $(2.5)$ is taken along any direction which is nontangent to the cut issuing from the point $\sqrt[\nu]{-\lambda_{1}}$. Here and henceforth the choice of the root is determined by the sector $\hat{S}$.

Proof. Let $y \in \Psi_{\hat{S}}$. Then Theorems $1.2,1.3$ and Corollary 2.1 imply

$$
F_{2}(y)-F_{1}(y)=\int_{\gamma} e^{-\lambda_{1} t^{\nu}} t^{-d} \tilde{f}_{(1)}(t, y) d t
$$

where the contour $\gamma$ consists of the part of $\ell_{1}$ from infinity to some fixed point $\xi_{1} \in \ell_{1}$, followed by a path from $\xi_{1}$ to some $\xi_{2} \in \ell_{2}$ and then along $\ell_{2}$ to infinity.

Now the assumption $F_{1}(y)=0$, according to (2.2) and (2.6), implies

$$
c=F_{2}(y)=c\left(\sqrt[\nu]{-\lambda_{1}}\right)
$$

where

$$
c(p)=\int_{\gamma} e^{p^{\nu} t^{\nu}} t^{-d} \tilde{f}_{(1)}(t, y) d t .
$$

On the other hand the assumption $F_{1}(y)=0$ implies $y \in \Psi_{\tilde{S}}$, where $\tilde{S} \supset \hat{S}$ and the opening of $\tilde{S}$ is greater than $\frac{\pi}{\nu}$. Then $y(x)$ satisfies the integral equation (1.6), where $\Gamma(x)=\Gamma_{\tilde{S}}(x)$ and $C=0$, and so

$$
x^{-d} y_{(1)}(x)=e^{\lambda_{1} x^{\nu}} \int_{\Gamma_{\tilde{s}}^{1}} e^{-\lambda_{1} t^{\nu}} t^{-d} \tilde{f}_{(1)}(t, y) d t .
$$

Applying the transformation $\mathcal{L}_{\nu}^{-1}$ in the sector $\tilde{S}$ we get after some calculations

$$
-\nu\left(p^{\nu}+\lambda_{1}\right) \stackrel{\circ}{Y}_{(1)}(p)=\mathcal{L}_{\nu}^{-1}\left[t^{-d-\nu+1} \tilde{f}_{(1)}(t, y)\right](p) .
$$

(For the sake of transparency in what follows we assume that $\hat{S}$ is bisected by the positive real semiaxis. Then $-\lambda_{1}>0$ and $\sqrt[\nu]{-\lambda_{1}}=\left|\lambda_{1}\right|^{1 / \nu}$.)

Theorem 2.1 asserts the holomorphy of the right hand side of (2.9) in some region, which contains the interval $\left(0, \sqrt[\nu]{-\lambda_{1}}\right)$. Let us show that for any $p_{0} \in\left[0, \sqrt[\nu]{-\lambda_{1}}\right]$

$$
\frac{2 \pi i}{\nu} \lim _{\substack{p \rightarrow p_{0} \\ \arg \left(p-p_{0}\right)=\beta}} \mathcal{L}_{\nu}^{-1}\left[t^{-d+1-\nu} \tilde{f}_{(1)}(t, y)\right](p)=c\left(p_{0}\right)
$$


where $\beta \in(0, \pi)$.

Indeed, the assumption $F_{1}(y)=0$ together with the properties $\left.\left.(1.5), \mathrm{b}\right)-\mathrm{d}\right)$ and Theorem 1.3 imply

$$
\tilde{f}(t, y)=O\left(x^{-2}\right), \quad x \longrightarrow \infty, x \in S \cup \tilde{S},
$$

where the closed sectorial region $S$ is bounded by the curve $\gamma$ and is situated on the right of $\gamma$.

Let us fix some $p$ which is sufficiently close to $p_{0}$ and with $\arg \left(p-p_{0}\right) \in(0, \pi)$. Let us divide the corresponding contour $\gamma_{0}$ from (2.4) into two parts $\gamma_{0}=\gamma_{0}^{+} \cup \gamma_{0}^{-}$, where $\gamma_{0}^{-} \subset \tilde{S} \backslash S, \gamma_{0}^{+} \subset S$ (here only the infinite parts of the contours are considered). Then, according to (2.11), the contours $\gamma$ and $\gamma_{0}$ of the integrals (2.8) and (2.9) can be deformed into $\delta=\gamma_{0}^{-} \cup \ell_{2}$, preserving the values of the integrals. Then (2.10) follows from the dominated convergence theorem.

Now, (2.7) - (2.10) and Theorem 2.1 imply

$$
c\left(p_{0}\right)=-2 \pi i\left(p_{0}^{\nu}+\lambda_{1}\right) \stackrel{\circ}{(1)}_{\left(p_{0}\right)}
$$

where $p_{0} \in\left[0, \sqrt[\nu]{-\lambda_{1}}\right)$. According to Remark 1.4 we can always assume that $\gamma \subset \hat{S}$. Then $\lim _{p_{0} \rightarrow \nu} \sqrt[\nu]{-\lambda_{1}} c\left(p_{0}\right)=c\left(\sqrt[\nu]{-\lambda_{1}}\right)$ follows from the inequality $\left|e^{-\lambda_{1} x^{\nu}}\right| \geq\left|e^{p_{0}^{\nu}} x^{\nu}\right|$ when $x \in \gamma$. Then (2.10) also holds when $\beta=\pi$.

We can now choose another $y \in \Psi_{\hat{S}}$ so that $F_{2}(y)=0$ and similarly show that $-F_{1}(y)=c\left(\sqrt[\nu]{-\lambda_{1}}\right)$ and that (2.10) holds for $\beta \in[\pi, 2 \pi)$. Then (2.5) follows from (2.7), (2.10).

Let us define the asymptotic difference $\Delta_{\hat{S}} y_{(1)}(x)$ of $y_{(1)}(x)$ in the sector $\hat{S}$ as the difference in asymptotics of $y_{(1)}(x)$ along the curves $\ell_{2}$ and $\ell_{1}$.

Corollary 2.2. For any $y \in \Psi_{\hat{S}}$ the asymptotic difference $\Delta_{\hat{S}} y_{(1)}(x)=e^{q_{1}(x)} c$. That is to say, the transition function $\varphi_{\hat{S}}(z)=z+c$.

Indeed, Corollary 2.2 follows from the fact that $F_{2}(y)=0$ implies $F_{1}(y)=-c$ (see the end of the proof of Theorem 2.2).

Remark 2.3. For any $d$ such that $\Re d \geq 0$, the limit $(2.10)$, where $\tilde{f}_{(1)}(t, y)$ is replaced by $\tilde{f}(t, y)$, exists and is equal to the corresponding constant (depending on $p_{0}$ ) vector.

\section{Evaluation of Stokes constants.}

3.1. Formulae for Stokes constants. Let $\hat{z}(x)$ be the formal solution of the original equation (0.4) and let $\hat{y}(x)$ be the formal solution of the corresponding prenormalized equation (1.5). Then

$$
\hat{z}(x)-\sum_{k=1}^{\eta} z_{k} x^{-k}=T(x) S(x) \hat{y}(x),
$$

where the number $\eta$ and the matrices $T(x)$ and $S(x)$ were defined in $\S 1$.

Let $y \in \Psi_{\hat{S}}$ be a solution of (1.5). Then

$$
z(x)=\sum_{k=1}^{\eta} z_{k} x^{-k}+T(x) S(x) y(x)
$$


is a proper solution of (0.4) admitting the asymptotics $\hat{z}(x)$ in $\hat{S}$. According to (2.1), the asymptotic difference

$$
\begin{aligned}
\Delta_{\hat{S}} z(x) & =T(x) S(x) e^{Q(x)}\left[\hat{F}_{2}(y)-\hat{F}_{1}(y)\right]+O\left(x^{-n(r+1)-\delta-1}\right) \\
& =c T^{(1)}(x) e^{\tilde{q}_{1}(x)}+O\left(x^{-n(r+1)-\delta-1}\right),
\end{aligned}
$$

where $c$ is the corresponding transition constant and $T^{(1)}(x)$ is the first column of $T(x)$. According to Remark 2.2,

$$
\tilde{q}_{1}(x)=q_{1}(x)-[n(r+1)+\delta] \ln x=\lambda_{1} x^{\nu}+b_{1} \ln x,
$$

where $b_{1}$ is defined in $\left.(1.5), \mathrm{e}\right)$. So

$$
\Delta_{\hat{S}} z(x)=(C+o(1)) x^{b_{1}} e^{\lambda_{1} x^{\nu}}+O\left(x^{-n(r+1)-\delta-1}\right),
$$

where $C=c T^{(1)}(\infty)=c h$, and $\|h\|=1$, see Theorem 1.1. Moreover, according to the assumption b) from the Introduction and to Corollary 1.1, both $b_{1}$ and $h$ are uniquely determined.

Definition 3.1. The vector $C=\operatorname{Col}\left(C_{1}, \ldots, C_{n}\right)$ is called the vector of Stokes constants of the solution $z(x)$ of $(0.4)$ in the sector $\hat{S}$.

According to (1.10)

$$
x^{b_{1}} e^{\lambda_{1} x^{\nu}}=O\left(x^{-n(r+1)-\delta-\mu}\right), \quad x \rightarrow \infty, x \in \ell_{1,2} .
$$

Since $\mu \in(0,1)$, the exponential term $C x^{b_{1}} e^{\lambda_{1} x^{\nu}}$ is the leading term of $\Delta_{\hat{S}} z(x)$ near the boundaries $\ell_{1,2}$ of $S$ as $x \rightarrow \infty$.

Remark 3.1. The change of variables $z(x) \mapsto z(x)+\sum_{k=1}^{N} z_{k} x^{-k}$, where $N \in \mathbb{N}$, in the equation (0.4) does not affect the exponential term $C x^{b_{1}} e^{\lambda_{1} x^{\nu}}$

For a scalar function $u(x)$, holomorphic in some sector $\tilde{S}$ of opening more than $\pi / \nu$, we introduce the operator

$$
\mathcal{R} u=-2 \pi i \lim _{p \rightarrow \sqrt[\nu]{-\lambda_{1}}}\left(p^{\nu}+\lambda_{1}\right)\left[\mathcal{L}_{\nu}^{-1} u\right](p)
$$

where the limit is taken in the sense of Theorem 2.2. It is clear that $\mathcal{R} y=0$ if $\mathcal{L}_{\nu}^{-1} y$ exists and is continuous at $p=\sqrt[\nu]{-\lambda_{1}}$.

Theorem 3.1. Let the characteristic exponent $e^{q_{1}(x)}$ be decreasing in the sector $\hat{S}$ of opening $\pi / \nu$. Then the vector of Stokes constants of the solution $z(x)$ in $\hat{S}$ is given by

$$
C=\mathcal{R}\left[x^{-b_{1}} z(x)\right]
$$

Remark 3.2. According to Remark 3.1, we can always assume the existence of $\mathcal{R}\left[x^{-b_{1}} z(x)\right]$

The following Lemmas precede the proof.

Lemma 3.1. If $\mathcal{R} y$ exists, then $\mathcal{R}\left(y / x^{\alpha}\right)=0$ for any $\alpha>0$. 
Proof. Assume that $\nu=1$. The existence of $\mathcal{R} y$ means

$$
Y(p)=\frac{\mathcal{R} y}{-2 \pi i}\left(p+\lambda_{1}\right)^{-1}+o\left(p+\lambda_{1}\right)^{-1}, \quad p \rightarrow-\lambda_{1},
$$

where $Y=\mathcal{L}_{\nu}^{-1} y$. Then we need to show

$$
\lim _{p+\lambda_{1} \rightarrow 0}\left(p+\lambda_{1}\right)\left[Y(p) * p^{\alpha-1}\right]=0 .
$$

Let us fix some small $\varepsilon \in \mathbb{C}$ and represent the convolution as

$$
Y(p) * p^{\alpha-1}=\left[\int_{0}^{p-\varepsilon}+\int_{p-\varepsilon}^{p}\right] Y(\tau)(p-\tau)^{\alpha-1} d t .
$$

It is easy to check that only the second integral is actually of interest here .

Without any loss of generality one can assume that $-\lambda_{1}, \varepsilon>0, p \in\left(0,-\lambda_{1}\right)$. Let $\delta=-\left(\lambda_{1}+p\right)>0$. Then in fact (3.7) follows from

$$
\lim _{\delta \rightarrow 0} \delta \int_{\delta}^{\delta+\varepsilon} \frac{\sigma^{\alpha-1}}{\delta+\sigma} d \sigma=0
$$

This assertion is a consequence of the estimates $2 \delta(\delta+\varepsilon)^{\alpha-1}|\ln 2 \delta|$ in the case $\alpha \geq 1$ and $2 \delta^{\alpha}|\ln 2 \delta|$ in the case $\alpha \in(0,1)$.

The case $\nu \neq 1$ can be reduced to the considered one.

Lemma 3.2. If $j \neq 1$, then $\mathcal{R} y_{(j)}=0$.

Proof. The solution $y \in \Psi_{\tilde{S}}$. Hence $y_{(j)}(x), j \in\{1, \ldots, n\}$, satisfies the integral equation

$$
y_{(j)}(x)=e^{\lambda_{j} x^{\nu}+\tilde{q}_{j}(x)} \int_{\Gamma_{\tilde{S}}^{j}(x)} e^{-\lambda_{j} t^{\nu}-\tilde{q}_{j}(t)} \tilde{f}_{(j)}(t, y) d t
$$

where $\tilde{q}_{j}(x)$ is a polynomial of degree less than $\nu$, which may also contain a logarithmic term. The equation (3.8) can be represented

$$
\begin{aligned}
y_{(j)}(x) & =e^{\lambda_{j} x^{\nu}+\tilde{q}_{j}(x)}\left(1+\frac{\tilde{q} \prime(x)}{\left(p^{\nu}+\lambda_{j}\right) x^{\nu-1}}\right) I(x)-e^{\lambda_{j} x^{\nu}+\tilde{q}_{j}(x)} \frac{\tilde{q}^{\prime}(x)}{\left(p^{\nu}+\lambda_{j}\right) x^{\nu-1}} I(x) \\
& =A_{1}(x)+A_{2}(x),
\end{aligned}
$$

where $I(x)$ denotes the integral in (3.8).

Now, by means of integration by parts we get

$$
\mathcal{L}_{\nu}^{-1} A_{1}=-\left(p^{\nu}+\lambda_{j}\right)^{-1} \mathcal{L}_{\nu}^{-1}\left[x^{1-\nu} \tilde{f}_{(j)}(x, y)\right]
$$

Then, according to Remark $2.3, j \neq 1$ implies $\mathcal{R} A_{1}=0$. The fact that $\mathcal{R} A_{2}=0$ follows from Lemma 3.1 since $\operatorname{deg} \tilde{q}^{\prime}(x)<\nu-1$. Finally, the remark $\mathcal{R} y_{(j)}=\mathcal{R} A_{1}+\mathcal{R} A_{2}=0$ completes the proof.

Proof of Theorem 3.1. Using Remark 3.1 and Lemma 3.2 we get from (3.2)

$$
\mathcal{R}\left[x^{-b_{1}} z(x)\right]=\mathcal{R}\left[x^{-b_{1}} T(x) S(x) y(x)\right]=\mathcal{R}\left[T^{(1)}(x) x^{-b_{1}-n(r+1)-\delta} y_{(1)}(x)\right] .
$$

Then, according to Remark 2.2, Lemma 3.1 and Theorem 2.2,

$$
\mathcal{R}\left[x^{-b_{1}} z(x)\right]=T^{(1)}(\infty) \mathcal{R}\left[x^{-d} y_{(1)}(x)\right]=C .
$$

Remark 3.3. By the same technique one can evaluate the higher order terms of $\Delta_{\hat{S}} z$. 
3.2. Stokes constants for Painleve II. Let us consider the well-known second Painleve equation

$$
v^{\prime \prime}(x)=2 v^{3}+x v+\alpha
$$

where $\alpha \in \mathbb{C}$ is a parameter. There exist three different formal power series solutions of (3.10). We consider here one of them, given by

$$
\hat{v}(x)=-\frac{\alpha}{x}+\frac{2 \alpha\left(\alpha^{2}-1\right)}{x^{4}}+\cdots=\frac{\alpha}{x} \sum_{k=0}^{\infty} v_{k} x^{-3 k}
$$

where the coefficients $v_{k}$ are polynomials in $\alpha$.

It is known (and one can easily check) that (3.10) is a one-level equation of order $\nu=3 / 2$, and that the linear part of (3.10), corresponding to the formal solution (3.11), is the well-known Airy equation $v^{\prime \prime}(x)=x v$. The Stokes rays of the Airy equation are given by

$$
\arg x=\frac{\pi}{3}+\frac{2}{3} \pi k, \quad k \in \mathbb{Z}
$$

The characteristic exponential $x^{-1 / 4} e^{-\frac{2}{3} x^{3 / 2}}$ of the Airy equation is decreasing in the sector $\hat{S}=\left\{x:|\arg x|<\frac{\pi}{3}\right\}$. Let $v \in \Psi_{\hat{S}}$. It is known (see, for instance, [T2], $\S 4.2$ ), that the exponential corrections of the asymptotics $\hat{v}(x)$ near the boundaries of $\hat{S}$ are proportional to the Airy function $A i(x)$, i.e. of the type $c x^{-1 / 4} e^{-2 / 3 x^{3 / 2}}$. Then, according to Theorem 3.1, the Stokes constant $c$ of the solution $v(x)$ in $\hat{S}$ is given by

$$
c=-2 \pi i \lim _{p \rightarrow\left(\frac{2}{3}\right)^{\frac{2}{3}}}\left(p^{\frac{3}{2}}-\frac{2}{3}\right) \mathcal{L}_{\frac{3}{2}}^{-1}\left[x^{\frac{1}{4}} v(x)\right](p) .
$$

Here $\mathcal{L}_{3 / 2}^{-1}\left[x^{-1 / 4} v(x)\right](p)$ is equal to the formal $\frac{3}{2}$-Borel transform $\mathcal{B}_{3 / 2}$ of the series $x^{1 / 4} \hat{v}(x)$, which is defined by

$$
\mathcal{B}_{3 / 2}\left(x^{-\alpha}\right)=\frac{p^{\alpha-\frac{3}{2}}}{\Gamma\left(\frac{2}{3} \alpha\right)}
$$

where $\Gamma$ denotes the gamma-function. (Equivalently we can suppose that $F_{1}(v)=0$ and evaluate $\mathcal{L}_{3 / 2}^{-1}\left[x^{-1 / 4} v(x)\right](p)$ in the corresponding sector.)

Then

$$
c=-2 \pi i \alpha \lim _{p \rightarrow\left(\frac{2}{3}\right)^{\frac{2}{3}}} p^{-\frac{3}{4}} W(p)
$$

where the power series $W(p)=\sum_{k=0}^{\infty} w_{k} p^{\frac{3}{2} k}$ has radius of convergence $\frac{2}{3}^{\frac{2}{3}}$. Here $w_{2 k}=-\frac{2}{3} \frac{v_{k}}{\Gamma\left(2 k+\frac{1}{2}\right)}, w_{2 k+1}=\frac{v_{k}}{\Gamma\left(2 k+\frac{1}{2}\right)}$. 
In the particular case $\alpha=0$ the Stokes constant $c=0$. This demonstrates that if a solution $v(x) \in \Psi_{\hat{S}}$ (i.e. if $v(x) \sim 0$ in $\hat{S}$ ), then it has the same exponential corrections on both rays $\arg x= \pm \frac{\pi}{3}$.

3.3. Other examples. The "asymptotics beyond all orders" approach, currently developed by M. D. Kruskal and H. Segur (see [KS]), yields a number of interesting problems requiring the calculation of Stokes constants. Let us return to the equation (0.1), which describes the trailing oscillations of the singularly perturbed fifth order Korteweg de-Vries equation ([GJ]).

The equation $(0.1)$ is a one-level equation of order 1 , which have characteristic exponentials $e^{ \pm i x}$. This equation possesses a formal power series solution

$$
\hat{v}(x)=-\frac{6}{x^{2}}+\frac{90}{x^{4}}-\cdots=\sum_{k=1}^{\infty} v_{k} x^{-2 k} .
$$

Let

$$
v(x) \sim \hat{v}(x), \quad x \longrightarrow \infty, \quad|\arg x|<\pi .
$$

What are the exponential corrections to the asymptotics (3.13) with respect to the rays $\arg x= \pm \pi$ ? It can be shown directly that they are of the form $c_{ \pm} e^{ \pm i x}$ (see also [GJ]), so according to Theorem 3.1

$$
c_{ \pm}=-2 \pi i \lim _{p \rightarrow \pm i}(p \pm i) V(p)
$$

where $V(p)=\sum_{k=1}^{\infty} \frac{v_{k}}{(2 k-1) !} p^{2 k-1}$. It is easy to check that $c_{+}=c_{-}$and $c_{+}$is a purely imaginary number. The evaluation of $c_{ \pm}$is discussed in [GJ].

\section{References}

[BJL] W. Balser, J. B. Jurkat, and D. A. Lutz, A general theory of invariants for meromorphic linear differential equations. Part I, Formal invariants, Funkcial. Ekvac. 22 (1979), 197-221.

[Fr] S. Friedland, On pointwise and analytic similarity of matrices, Israel J. Math. 35 (1980), 89-108.

[GJ] R. Grimshaw and N. Joshi, Weakly non-local solitary waves in a singularly perturbed Kortewegde Vries equation, SIAM J. Appl. Math., submitted.

[GM] V. Gurariı and V. Matsaev, Stokes multipliers for systems of linear ordinary differential equations of first order, Soviet Math. Dokl. (1) 31 (1985), 52-56.

[Gr] R. Grimshaw, The use of Borel-summation in the establishment of non-existence of certain travelling-wave solutions of the Kuramoto-Sivashinsky equations, Wave Motion 15 (1992), 393-395.

[HM] V. Hakim and K. Mallick, Exponentially small splitting of separatrices, matching in the complex plane and Borel summation, Nonlinearity (1) 6 (1993), 57-70.

[KS] M. D. Kruskal and H. Segur, Asymptotics beyond all orders in a model of crystal growth, Studies in Applied Math. (2) 85 (1991), 129-181.

[KT] S. G. Krein and A. Tovbis, Linear singular differential equations in finite-dimensional and Banach spaces, Leningrad Math. J. (5) 2 (1991), 931-985.

[PRG] Y. Pomeau, A. Ramani, and B. Grammaticos, Structural stability of the Korteweg-de Vries solutions under a singular perturbation, Physica D 31 (1988), 127-134.

[RS] J. P. Ramis and Y. Sibuya, Hukuhara domains and fundamental existence and uniqueness theorems for asymptotic solutions of Gevrey type, Asymptotic Analysis 2 (1989), 39-94. 
[T1] A. Tovbis, Normal forms of holomorphic matrix-valued functions and corresponding forms for singular differential operators, Linear Algebra and Applications 162-164 (1992), 389-407.

[T2] - Nonlinear ordinary differential equations resolvable with respect to an irregular singular point, J. of Differential Equations (1) 107 (1994), to appear.

[T3] _ Asymptotics beyond all orders and analytic properties of inverse Laplace transforms of solutions, Comm. Math. Phys., to appear.

[Wa] W. Wasow, Asymptotic Expansions for Ordinary Differential Equations, Dover, N. Y., 1976.

Department of Theoretical Mathematics, The Weizmann Institute of Science, Rehovot 76100, ISRAEL 